\section{Immunotherapy of sepsis}

Septicaemia continues to have a poor prognosis, with an overall mortality of $20-60 \%$, those who develop septic shock faring worse. ${ }^{1,2}$ This is despite significant improvements in intensive care management over the last ten years, and the development of new and more effective antibiotics. Endotoxin has long been recognised as the main toxic bacterial product in infections with gram-negative bacteria, able to reproduce in experimental animals and man many of the features of gram-negative septicaemia and shock. Endotoxin produces these effects through several different pathways, but recently it has become clear that the induction by endotoxin of the synthesis of cytokine mediators is central to the pathophysiology of gram-negative sepsis. ${ }^{3}$ The most important of these cytokines are tumour necrosis factor (TNF) and interleukin (IL)-1 which in animals are able to reproduce the tissue damage and multi-organ failure characteristic of sepsis. Antibodies to TNF are protective in animal models of sepsis, and TNF levels correlate with severity of disease in meningococcal septicaemia in man. ${ }^{4,5}$ These cytokines are also produced in infections with gram-positive bacteria and thus may represent a final common pathway by which infection with various microorganisms produces septic shock and death (figure).

There are a number of points in these pathways for potential therapeutic intervention. Firstly, antibodies and other agents that neutralise endotoxin might be of benefit in the treatment of gram-negative sepsis. In addition, agents that neutralise the effects of TNF or IL-1 may also arrest the development of septic shock and have the added benefit of potential use in infection with both gram-negative and gram-positive bacteria. Several agents are undergoing clinical trials, most of which are not yet finished, so that our understanding of their potential therapeutic role is necessarily incomplete.

\section{Antibodies to endotoxin}

Polyclonal antibodies against endotoxin have been used in the treatment of sepsis in several trials; the antisera used have been raised against mutant endotoxins which lack the hypervariable $\mathrm{O}$ side-chain, thus producing antibodies that react with the relatively invariant core polysaccharide and lipid A components of endotoxin, which differ little between different gram-negative organisms. Therefore, these antibodies might offer protection against infections with a range of gram-negative bacteria. Initial studies showed some evidence of a protective effect in treating gramnegative bacteraemia, ${ }^{6}$ although further studies have

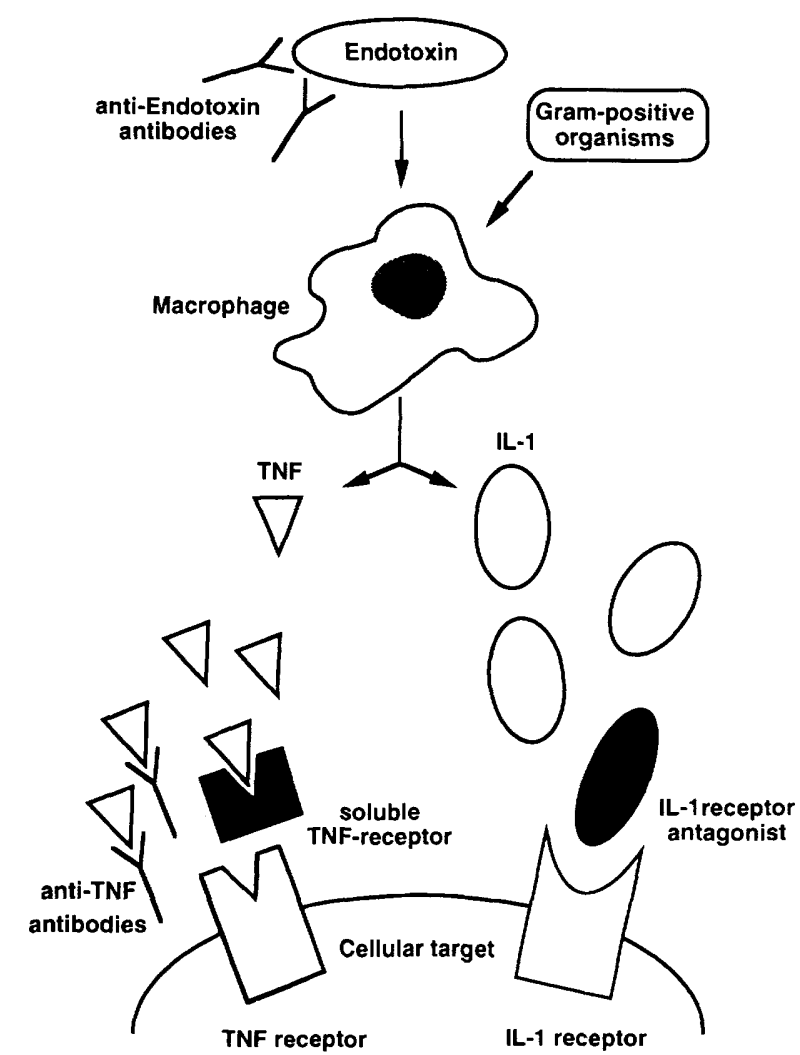

Figure. Cytokine pathways in sepsis. TNF, Tumour necrosis factor: IL-1, Interleukin 1 .

not always shown any benefit. ${ }^{7}$ These polyclonal antisera have also been used prophylactically in patients at high risk of developing gram-negative septicaemia; again, earlier promising beneficial effects ${ }^{8}$ have not been found in more recent studies. ${ }^{9}$ However, in this latter study, ${ }^{9}$ some benefit in preventing the incidence of infection was seen with a standard immune globulin preparation, which had a much lower titre of anti-endotoxin antibody than the serum selected for anti-core lipopolysaccharide activity. Because of these variable results with polyclonal antisera, several monoclonal antibodies (MAbs) that bind to the core and lipid $A$ region of endotoxin have been generated. One of these, HA-1A (Centoxin), a human IgM MAb, is currently licensed for use in several European countries. In a double-blind, placebo-controlled trial of HA-1A in the treatment of patients with the sepsis syndrome, no benefit was seen in 28-day mortality in the whole group of 543 patients treated, but there was a significant reduction in 28-day mortality from $49 \%$ in the placebo-treated group to $30 \%$ in the patients who received HA-1A, in a sub-group of 200 patients who subsequently proved to have gramnegative bacteraemia. ${ }^{2}$ Since an anti-endotoxin can 
only be effective in infections with gram-negative bacteria, particularly when associated with elevated plasma endotoxin levels, this is clearly the group one would expect to benefit from this agent; moreover, the more severe the septic shock, the more marked was the protection seen. Thus, in a subgroup of 102 patients with shock on admission, the mortality at 28 days was reduced from $57 \%$ in the placebo-treated group to $33 \%$ in the HA-1A-treated patients.

However, there is a great deal of controversy over the use of HA-1A. In the clinical trial described above, the statistical analysis has been criticised because of inappropriate corrections for multiple subgroups and end points. ${ }^{10}$ Furthermore, these authors are concerned that the protective effect was seen only at centres with high mortality, that the patient stratification for severity of illness was inappropriately applied, and that patients who received inadequate or unknown antibiotic treatment were included in the analysis, potentially masking significant differences between the placebo and HA-1A-treated groups. It has emerged that, during the study, an additional end point of 28-day mortality was added to the 14-day mortality end point in the original protocol; benefit was seen only in the 28-day mortality group. Results of interim analysis were available to the manufacturers prior to submission of the revised protocol, thus causing concern that there may have been potential bias in selecting an end point for the trial. Because of these concerns, the United States Food and Drug Administration refused licensing of HA-1A. ${ }^{11}$ Further placebo-controlled trials in sepsis and meningococcal disease are now in progress and it is to be hoped that they will help to clarify the situation.

The mode of action of this MAb is still uncertain. Some groups report little or no binding to heterologous smooth gram-negative organisms, and the original animal experiments suggesting a protective effect in sepsis have been difficult to reproduce. ${ }^{12}$ It is also difficult to demonstrate neutralising ability of this antibody in vitro. Various explanations for these discrepancies have been advanced. One possibility is that HA-1A mediates removal of endotoxin by adherence to red blood cells via the CR 1 receptor; the red cell-antibody-endotoxin complexes are then cleared by the reticuloendothelial system. Although there is some evidence that HA-1A-endotoxin complexes can bind to red cells via $\mathrm{CR} 1$ in vitro, ${ }^{13}$ there is as yet no evidence that this mechanism operates in vivo.

A second MAb to the lipid A region of endotoxin, known as E5, has also undergone clinical trials. This is a murine IgM MAb which has been tested in two placebo-controlled trials. The results of the first trial suggested that there was a significant benefit only in a subgroup of patients with gram-negative sepsis who were not in refractory shock; a second trial designed to test the hypothesis that E5 would be of benefit in this subgroup did not confirm this finding but did show a trend to improved survival in further subgroup of patients with major-organ failure without refractory shock. ${ }^{14}$ This MAb has not yet been approved for clinical use.

\section{Cytokines as targets}

Several agents that block the action of cytokines have been developed. MAbs to TNF have been shown to be effective in protecting against mortality in various animals models-indeed this is evidence for the importance of TNF in mediating the lethality of overwhelming sepsis. ${ }^{3}$ However, blocking the effects of TNF action is not universally effective in reducing mortality. In localised infection such as peritonitis, some experimental models have shown an increased mortality after administration of anti-TNF, arguing for a protective role for TNF in these circumstances. ${ }^{15}$ A number of anti-TNF antibodies are in clinical trial at present, and the results of these investigations are awaited with interest, since promising results of a drug in experimental models do not always translate into clinical efficacy. One multicentre placebo-controlled trial of an anti-TNF MAb was terminated recently because of a trend towards a higher mortality in a treated group of patients who were not in shock at entry to the trial; the reason for this is as yet unknown.

In addition to antibodies, the discovery of natural antagonists of cytokine action has led to further therapeutic possibilities. These include a soluble form of the TNF-receptor and a competitive inhibitor of IL1 binding, the IL- 1 receptor antagonist, IL-1Ra. IL$1 \mathrm{Ra}$ appears to be a particularly promising agent, highly effective in reducing mortality in a number of experimental models of infection. Preliminary results of its efficacy in phase II trials suggest a dosedependent improvement in mortality due to sepsis. ${ }^{16} \mathrm{~A}$ large phase III trial of IL-1Ra in the sepsis syndrome is currently in progress.

\section{Current use of immunotherapy}

A range of other novel therapies for septic shock are also under investigation, and undoubtedly over the next few years results of clinical trials with these reagents will appear. At present, however, the only agent licensed for clinical use is the anti-endotoxin MAb, HA-1A. What recommendations can be made concerning its use? As detailed above, the evidence of its effectiveness in the treatment of gram-negative sepsis is far from clear cut. Some hospitals have chosen to await the results of further trials before adding HA$1 \mathrm{~A}$ to their formulary. Its side effects are minimal but at a cost of $£ 2,200$ per dose, its use must be carefully monitored. One approach would be to restrict its use to those patients who have a high probability of a gram-negative bacteraemia and who are likely to deteriorate rapidly; this would include patients with gram-negative pneumonia, gastrointestinal sepsis, or an unknown source of infection. Pregnant women, and patients with burns or neutropenia should be excluded, as they were not included in the patient 
groups treated in the clinical trial. Children with meningococcal disease present a particularly difficult dilemma, since many believe that there is a high likelihood that they will respond to HA-1A. However, this group was excluded from the original pivotal trial, so there is no formal proof of efficacy. For the time being, such patients should be considered for entry into a placebo-controlled trial which is in progress. ${ }^{17}$ Many units have established an ad hoc group of intensivists and infectious diseases physicians or microbiologists to oversee the use of the drug, but clinical diagnostic accuracy of gram-negative bacteraemia is poor, about $50 \%$ at best. There is some evidence to suggest that delayed treatment with HA-1A may reduce its potential benefit considerably, so any control over its prescription needs to be flexible. The

\section{References}

1. Roberts FJ, Geere IW, Coldman A. A three-year study of positive blood cultures, with emphasis on prognosis. $J$ Infect Dis $1991 ; 13: 3446$.

2. Ziegler EJ, Fisher CJ Jr, Strung CL et al. Treatment of gramnegative bacteremia and septic shock with HA-1A human monoclonal antibody against endotoxin-a randomized, double-blind, placebo-controlled trial. N Engl J Med 1991; 324: 429-36.

3. Glauser MP, Zanetti G, Baumgartner J-D, Cohen J. Septic shock: pathogenesis. Lancet $1992 ;$ 338: 732-6.

4. Tracey KJ, Fong Y, Hesse DG et al. Anti-cachectin/tumour necrosis factor antibodies prevent septic shock during lethal bacteraemia. Nature 1987 ; 330: 662-4.

5. Waage A, Halstensen A, Espevik T. Association between tumour necrosis factor in serum and fatal outcome in patients with meningococcal disease. Lancet 1987; i: 355-7.

6. Ziegler EJ, McCutchan JA, Fierer $\mathbf{J}$ et al. Treatment of gramnegative bacteremia and shock with human antiserum to a mutant Escherichia coli. N Engl J Med 1982; 307: 1225-30.

7. Baumgartner JD, Glauser MP. Controversies in the use of passive immunotherapy for bacterial infections in the critically ill patient. Rev Infect Dis 1987; 9: 194-205.

8. Baumgartner JD, Glauser MP, McCutchan JA et al. Prevention of gram-negative shock and death in surgical patients by antibody to endotoxin core glycolipid. Lancet $1985 ; 2$. 59-63.

9. The Intravenous Immunoglobulin Study Group. Prophylactic administration of standard immune globulin as compared with core-lipopolysaccharide immune globulin in patients at high risk of postsurgical infection. N Engl J Med 1992; 327: $234-40$. results of further trials with HA-1A and with the other agents described here will soon become available, and it is to be hoped that these data will clarify a difficult and confused situation and that an effective therapy will emerge which, together with antibiotic treatment, will reduce the mortality of septicaemia.

T. J. Evans and J. COHEN

Department of Infectious Diseases and Bacteriology, Royal Postgraduate Medical School, Du Cane Road, London W12 0NN.

TJE is supported by an MRC Clinician/Scientist Research Fellowship.

Since this article was written, preliminary results of a second trial of HA-1A (Centoxin) showed an excess mortality in treated patients with non-gram negative bacteraemia. Because of this as yet unexplained finding, the manufacturers of HA-1A have for the present withdrawn it from sale.

10. Warren HS, Danner RL, Munford RS. Sounding aboard: antiendotoxin antibodies. $N$ Engl J Med 1992; 326: 1153-7.

11. Siegel JP, Stein KE, Zoon KC. Anti-endotoxin monoclonal antibodies (letter). $N$ Engl J Med 1992; 327: 890-1.

12. Baumgartner JD, Heumann D, Gerain J, Weinbreck P, Grau GE, Glauser MP. Association between protective efficacy of anti-lipopolysaccharide (LPS) antibodies and suppression of LPS-induced tumour necrosis $\alpha$ and interleukin 6. J Exp Med 1990; 171: 889-96.

13. Tonoli M, Davies KA, Appelmelk BJ, Walport MJ, Cohen J. In vitro binding of human monoclonal anti-lipid $A$ antibody (HA-1A) to gram-negative bacteria. Program and abstracts of the 32nd Interscience Conference on Antimicrobial Agents and Chemotherapy 1992; Abstract No. 10.

14. Wenzel R, Bone R, Fein A et al. Results of a second doubleblind randomized controlled trial of anti-endotoxin antibody E5 in gram-negative sepsis. Program and abstracts of the 31st Interscience Conference on Antimicrobial Agents and Chemotherapy 1991; Abstract No. 1170.

15. Echtenacher B, Falk W, Männel DN, Krammer PH. Requirement of endogenous tumour necrosis factor/cachectin for recovery from experimental peritonitis J Immunol 1990; 145: 3762-6.

16. Opal SM, Fisher CJ, Slotman GJ et al. The phase II Interleukin1 receptor antagonist (IL-1ra) sepsis syndrome trial: analysis of clinical, cytokine, and microbial features with outcome. Program and abstracts of the 32nd Interscience Conference on Antimicrobial Agents and Chemotherapy 1992; Abstract No. 1570.

17. Nadel S, Klein N, Heyderman R, Levin M. Endotoxin antibody for sepsis in infants. Lancet 1992; 339: 678. 